International Mathematical Forum, 2, 2007, no. 52, 2579 - 2586

\title{
Finite Rings and Loop Rings \\ Involving the Commuting Regular Elements
}

\author{
H. Doostie \\ Mathematics Department \\ Teacher Training University \\ 49 Mofateh Ave., Tehran 15614, Iran \\ doostih@saba.tmu.ac.ir \\ L. Pourfaraj \\ Department of Mathematics \\ Science and Research Branch \\ Islamic Azad University \\ P. O. Box 14515/1775, Tehran, Iran \\ L.pourfaraj@iauctb.ac.ir
}

\begin{abstract}
Two elements $x$ and $y$ of a ring $R$ are commuting regular if for some $a \in R, x y=y x a y x$ holds. In this paper we study the finite rings $Z_{p}[S]$ and $Z_{p_{1}^{i} p_{2}}\left[L_{n}(m)\right]$, and prove that the first one is commuting regular and the second ring contains the commuting regular element and idempotents as well (where $p, p_{1}$ and $p_{2}$ are odd primes. Moreover, $i, m$ and $n$ are positive integers such that $m<n,(m, n)=1$ and $(m-1, n)=1)$.
\end{abstract}

Mathematics Subject Classification: 16E50, 12E15, 16N60

Keywords: Commuting regular rings, group rings, loop ring

\section{Introduction}

We use $R$ and $S$ to denote a ring and a semigroup, respectively. A quasi group is a set $Q$ with a binary operation, here denoted by ".", with the property that for all $a, b \in Q$, there are unique solutions to the equations $a . x=b$ and $y . a=b$. A quasi group with an identity element is called a loop. A ring $R$ is called commuting regular if and only if for each $x, y \in R$ there exists an element $a$ of $R$ such that $x y=y x a y x$ (see [6]). The commuting regular semigroup is defined 
in a similar way in [2]. A positive integer $n$ is said to be a perfect number if $n$ is equal to the sum of all its positive divisors, excluding $n$ itself (see [1]). Let $R$ be a ring, $G$ is a group and $R[G]$ be the set of all linear combinations of the form $\alpha=\sum_{g \in G} \alpha(g) g$ where $\alpha(g) \in R$ and $\alpha(g)=0$ except of a finite number of coefficients. The sum and product of elements of $R[G]$ are defined by:

$$
\begin{aligned}
\left(\sum_{g \in G} \alpha(g) g\right)+\left(\sum_{g \in G} \beta(g) g\right) & =\sum_{g \in G}(\alpha(g)+\beta(g)) g, \\
\left(\sum_{g \in G} \alpha(g) g\right)\left(\sum_{h \in G} \beta(h) h\right) & =\sum_{g, h \in G} \alpha(g) \beta(h) g h .
\end{aligned}
$$

$R[G]$ is called the group ring of $G$ over $R$ (see [4]). If we replace the group $G$ in the above definition by a semigroup $S$ (or loop $L$ ) we get $R[S]$ (or $R[L]$ ) the semigroup ring (or loop ring). Following [6], let $L_{n}(m)=\{e, 1,2, \ldots, n\}$ be a set where $n>3, n$ is an odd integer and $m$ is a positive integer such that $(m, n)=1$ and $(m-1, n)=1$ with $m<n$. Define on $L_{n}(m)$, a binary operation "." as follows:

(1) $e . i=i . e=i$ for all $i \in L_{n}(m)-\{e\}$,

(2) $i^{2}=e$ for all $i \in L_{n}(m)$,

(3) $i . j=t$ where $t \equiv(m j-(m-1) i)(\bmod n)$ for all $i, j \in L_{n}(m), i \neq e$ and $j \neq e$.

Then $L_{n}(m)$ is a loop.

\section{The commuting regular semigroup ring $Z_{p}[S]$}

Definition 2.1. A group ring $R[G]$ is said to be a commuting regular group ring if $R$ be a commuting regular ring. Also, we define the commuting regular semigroup ring, commuting regular loop ring and commuting regular groupoid ring in the same way.

Definition 2.2. Two elements $x$ and $y$ of a ring $R$ (or semigroup $S$ ) are commuting regular if for some $a \in R$ (or $a \in S$ ), xy=yxayx.

Proposition 2.3. Let $S=\{a, b, c\}$ be the semigroup given by the table,

\begin{tabular}{c|ccc} 
& $\mathrm{a}$ & $\mathrm{b}$ & $\mathrm{c}$ \\
\hline $\mathrm{a}$ & $\mathrm{a}$ & $\mathrm{a}$ & $\mathrm{a}$ \\
$\mathrm{b}$ & $\mathrm{a}$ & $\mathrm{b}$ & $\mathrm{a}$ \\
$\mathrm{c}$ & $\mathrm{a}$ & $\mathrm{a}$ & $\mathrm{c}$
\end{tabular}

then for all prime $p, Z_{p}[S]$ is commuting regular semigroup ring. 
Proof. If $p=2, Z_{2}[S]$ is a Boolean ring and so $Z_{2}[S]$ is a commuting regular semigroup ring. Now, let $p$ be an odd prime, then $2^{p} \equiv 2(\bmod p)$ and so

$$
(\alpha a+\beta b+\gamma c)^{p} \equiv(\alpha a+\beta b+\gamma c)(\bmod p),
$$

where $\alpha, \beta, \gamma \in Z_{p}$. Therefore $x^{p}=x$ for all $x \in Z_{p}[S]$ and so

$$
x y=x^{p} y^{p}=(y x)\left(x^{p-2} y^{p-2}\right)(y x)
$$

for all $x, y \in Z_{p}[S]$. Then $Z_{p}[S]$ is commuting regular semigroup ring.

Corollary 2.4. Let $S=\{a, b, c\}$ be the semigroup given by the table,

\begin{tabular}{c|ccc} 
& $a$ & $b$ & $c$ \\
\hline$a$ & $a$ & $a$ & $a$ \\
$b$ & $a$ & $b$ & $a$ \\
$c$ & $a$ & $a$ & $c$
\end{tabular}

then $R=\prod_{i \in I} Z_{p_{i}}$ is a commuting regular ring where $p_{i}$ is a prime number for all $i$.

Proof. By the Proposition 3.1 of [2] and the Proposition 2.3.

Proposition 2.5. Let $S=\{a, b, c\}$ be the semigroup given by the table,

\begin{tabular}{c|ccc} 
& $\mathrm{a}$ & $\mathrm{b}$ & $\mathrm{c}$ \\
\hline $\mathrm{a}$ & $\mathrm{a}$ & $\mathrm{a}$ & $\mathrm{a}$ \\
$\mathrm{b}$ & $\mathrm{a}$ & $\mathrm{b}$ & $\mathrm{a}$ \\
$\mathrm{c}$ & $\mathrm{a}$ & $\mathrm{a}$ & $\mathrm{c}$
\end{tabular}

then

$$
I=\{0, a, b, c,(p-1) a+b,(p-1) a+c,(p-1) a+b+c,(p-2) a+b+c\},
$$

is the set of all idempotent elements of commuting regular semigroup ring $Z_{p}[S]$.

Proof. Assume that $x$ be an idempotent of $Z_{p}[S]$, then $x=(\alpha a+\beta b+\gamma c)$ where $\alpha, \beta, \gamma \in Z_{p}$. By $x^{2}=x$, we have $\left(\alpha^{2}+2 \alpha \beta+2 \alpha \gamma+2 \beta \gamma\right)=\alpha, \beta^{2}=\beta$ and $\gamma^{2}=\gamma$. Then $\beta, \gamma \in\{0,1\}$.

(1) If $\beta=\gamma=0, \alpha \in\{0,1\}$ and so $x=0$ or $x=a$,

(2) If $\beta=0$ and $\gamma=1, \alpha \in\{0,(p-1)\}$ and so $x=c$ or $x=(p-1) a+c$,

(3) If $\beta=\gamma=1, \alpha \in\{(p-1),(p-2)\}$ and so $x=(p-1) a+b+c$ or $x=(p-2) a+b+c$. 
Example 2.6. Let $M=\{a, b, c\}$ be the groupoid given by the table,

\begin{tabular}{c|ccc} 
& $\mathrm{a}$ & $\mathrm{b}$ & $\mathrm{c}$ \\
\hline $\mathrm{a}$ & $\mathrm{a}$ & $\mathrm{b}$ & $\mathrm{c}$ \\
$\mathrm{b}$ & $\mathrm{c}$ & $\mathrm{a}$ & $\mathrm{b}$ \\
$\mathrm{c}$ & $\mathrm{b}$ & $\mathrm{c}$ & $\mathrm{a}$
\end{tabular}

then $Z_{2}[M]$ is the commuting regular groupoid ring having only 8 elements given by

$$
\{0, a, b, c, a+b, a+c, b+c, a+b+c\} .
$$

Clearly, $Z_{2}[M]$ is a non associative ring without identity and non commuting regular ring. But center of $Z_{2}[M]$ (i.e; $Z\left(Z_{2}[M]\right)=\{0, a+b+c\}$ )is commuting regular ring.

\section{The loop ring $Z_{p_{1}^{i} p_{2}}\left[L_{n}(m)\right]$}

In this section we will prove that existence of commuting regular elements for the loop ring $Z_{t}\left[L_{n}(m)\right]$ when $t$ is an even perfect number. Also we will prove that the loop ring $Z_{t}\left[L_{n}(m)\right]$ have commuting regular elements when $t$ is of the form $2^{i} p$ or $3^{i} p$ (where $p$ is an odd prime) or in general when $t=p_{1}^{i} p_{2}\left(p_{1}\right.$ and $p_{2}$ are distinct odd primes).

Proposition 3.1. Let $Z_{t}\left[L_{n}(m)\right]$ be a loop ring where $t$ is an even perfect number of the form $t=2^{r}\left(2^{r+1}-1\right)$ for some $r>1$, then there exists an idempotent element $e \in Z_{t}\left[L_{n}(m)\right]$ such that $e \neq 0,1$.

Proof. As $t$ be an even perfect number, $t$ must be of the form

$$
t=2^{r}\left(2^{r+1}-1\right), \text { for some } r>1
$$

where $\left(2^{r+1}-1\right)$ is a prime. Consider $e=2^{r}(1+l) \in Z_{t}\left[L_{n}(m)\right]$ where $l \in L_{n}(m)$. Now

$$
e^{2}=\left(2^{r}(1+l)\right)^{2}=2.2^{2 r}(1+l)
$$

by $2^{r} 2^{r+1} \equiv 2^{r}(\bmod t)$. Therefore $e^{2}=e$.

Example 3.2. The loop ring $Z_{6}\left[L_{n}(m)\right]$ has an idempotent $e=2(1+l)$ where $l \in L_{n}(m)$.

Proposition 3.3. Let $Z_{t}\left[L_{n}(m)\right]$ be a loop ring where $t$ is an even perfect number of the form $t=2^{r}\left(2^{r+1}-1\right)$ for some $r>1$, then there exist commuting regular elements $a, b \in Z_{t}\left[L_{n}(m)\right]$ such that $a \neq b$. 
Proof. As $t$ be an even perfect number, $t$ must be of the form

$$
t=2^{r}\left(2^{r+1}-1\right), \text { for some } r>1
$$

where $\left(2^{r+1}-1\right)$ is a prime. Assume that $a=2^{r}(1+l)$ and $b=\left(t-2^{r}\right)(1+l) \in$ $Z_{t}\left[L_{n}(m)\right]$. Now

$$
b^{2}=\left[\left(t-2^{r}\right)(1+l)\right]^{2}=\left(t-2^{r}\right)^{2}(1+l) \equiv 2^{r}(1+l)(\bmod t)
$$

by $2^{r} 2^{r+1} \equiv 2^{r}(\bmod t)$, so $b^{2}=a$. Also,

$$
a b=\left[2^{r}(1+l)\right]\left[\left(t-2^{r}\right)(1+l)\right] \equiv-2.2^{r} \cdot 2^{r}(1+l)(\bmod t)
$$

by $-2.2^{r} .2^{r}(1+l) \equiv\left(t-2^{r}\right)(1+l)(\bmod t)$ and so $a b=b$. Similarly, $b a=b$. By the Proposition 3.1, $a^{2}=a$. Therefore

$$
a b=(b a) b(b a)
$$

Example 3.4. The loop ring $Z_{6}\left[L_{n}(m)\right]$ have commuting regular elements $a=2(1+l)$ and $b=(6-2)(1+l)$ where $l \in L_{n}(m)$.

Proposition 3.5. Let $Z_{2 p}\left[L_{n}(m)\right]$ be a loop ring where $p$ is an odd prime such that $p \mid 2^{r+1}-1$ for some $r \geq 1$, then there exists an idempotent element $e \in Z_{2 p}\left[L_{n}(m)\right]$ such that $e \neq 0,1$.

Proof. Suppose that $p \mid 2^{r+1}-1$ for some $r \geq 1$ and $e=2^{r}(1+l) \in$ $Z_{2 p}\left[L_{n}(m)\right]$. Therefore

$$
e^{2}=\left(2^{r}(1+l)\right)^{2}=2 \cdot 2^{2 r}(1+l)=2^{r+1} \cdot 2^{r}(1+l) \equiv 2^{r}(1+l)(\bmod 2 p)
$$

by $2^{r} 2^{r+1} \equiv 2^{r}(\bmod 2 p)$, so $e^{2}=e$.

Example 3.6. The loop ring $Z_{10}\left[L_{n}(m)\right]$ has an idempotent $e=2^{3}(1+l)$ where $r=3,5 \mid 2^{3+1}-1$ and $l \in L_{n}(m)$.

Proposition 3.7. Let $Z_{2 p}\left[L_{n}(m)\right]$ be a loop ring where $p$ is an odd prime such that $p \mid 2^{r+1}-1$ for some $r \geq 1$, then there exist commuting regular elements $a, b \in Z_{2 p}\left[L_{n}(m)\right]$ such that $a \neq b$. 
Proof. Suppose that $p \mid 2^{r+1}-1$ for some $r \geq 1$ and $a=2^{r}(1+l), b=$ $\left(2 p-2^{r}\right)(1+l) \in Z_{2 p}\left[L_{n}(m)\right]$. Therefore

$$
b^{2}=\left[\left(2 p-2^{r}\right)(1+l)\right]^{2}=2\left(2 p-2^{r}\right)^{2}(1+l) \equiv 2.2^{2 r}(1+l)(\bmod 2 p)
$$

and

$$
2^{r+1} 2^{r}(1+l) \equiv 2^{r}(1+l)(\bmod 2 p)
$$

by $2^{r} 2^{r+1} \equiv 2^{r}(\bmod 2 p)$ and so $b^{2}=a$. Also,

$$
a b=\left[2^{r}(1+l)\right]\left[\left(2 p-2^{r}\right)(1+l)\right] \equiv-2^{r}(1+l) 2^{r}(1+l)(\bmod 2 p)
$$

and

$$
-2.2^{2 r}(1+l) \equiv\left(2 p-2^{r}\right)(1+l)(\bmod 2 p) .
$$

Hence $a b=b$. Similarly, $b a=b$. By the Proposition 3.6, $a^{2}=a$. Therefore

$$
a b=(b a) b(b a) \text {. }
$$

Example 3.8. The loop ring $Z_{10}\left[L_{n}(m)\right]$ have commuting regular elements $a=2^{3}(1+l)$ and $b=2(1+l)$ where $l \in L_{n}(m)$.

Proposition 3.9. Let $Z_{2^{i} p}\left[L_{n}(m)\right]$ be a loop ring where $p$ is an odd prime such that $p \mid 2^{r+1}-1$ for some $r \geq i$, then there exists an idempotent element $e \in Z_{2^{i} p}\left[L_{n}(m)\right]$ such that $e \neq 0,1$.

Proof. Suppose that $p \mid 2^{r+1}-1$ for some $r \geq i$ and $e=2^{r}(1+l) \in$ $Z_{2^{i} p}\left[L_{n}(m)\right]$. Since

$2^{r+1} \equiv 1(\bmod p)$ for some $r \geq i \Leftrightarrow 2^{r} .2^{r+1} \equiv 2^{r}\left(\bmod 2^{i} p\right)$ as $\left(2^{r}, 2^{i} p\right)=2^{i}, r \geq i$

then $e^{2}=e$.

Example 3.10. The loop ring $Z_{2^{3} .7}\left[L_{n}(m)\right]$ has an idempotent $e=2^{5}(1+l)$ where $r=5,7 \mid 2^{5+1}-1$ and where $l \in L_{n}(m)$.

Proposition 3.11. Let $Z_{2^{i} p}\left[L_{n}(m)\right]$ be a loop ring where $p$ is an odd prime such that $p \mid 2^{r+1}-1$ for some $r \geq 1$, then there exist commuting regular elements $a, b \in Z_{2^{i} p}\left[L_{n}(m)\right]$ such that $a \neq b$.

Proof. Suppose that $p \mid 2^{r+1}-1$ for some $r \geq 1$ and $a=2^{r}(1+l), b=\left(2^{i} p-\right.$ $\left.2^{r}\right)(1+l) \in Z_{2^{i} p}\left[L_{n}(m)\right]$. Since $2^{r} \cdot 2^{r+1} \equiv 2^{r}\left(\bmod 2^{i} p\right)$ as $\left(2^{r}, 2^{i} p\right)=2^{i}, r \geq i$,

$$
b^{2}=a \text { and } a b=b a=b .
$$

By the Proposition 3.9, $a^{2}=a$. Therefore

$$
a b=(b a) b(b a)
$$


Example 3.12. The loop ring $Z_{2^{3} .7}\left[L_{n}(m)\right]$ have commuting regular elements $a=2^{5}(1+l)$ and $b=\left(2^{3} .7-2^{5}\right)(1+l)$ where $r=5,7 \mid 2^{5+1}-1$ and $l \in L_{n}(m)$.

Proposition 3.13. Let $Z_{3^{i} p}\left[L_{n}(m)\right]$ be a loop ring where $p$ is an odd prime such that $p \mid 2.3^{r}-1$ for some $r \geq i$, then there exists an idempotent element $e \in Z_{3^{i} p}\left[L_{n}(m)\right]$ such that $e \neq 0,1$.

Proof. Suppose that $p \mid 2.3^{r}-1$ for some $r \geq i$ and $e=3^{r}(1+l) \in$ $Z_{3^{i} p}\left[L_{n}(m)\right]$. Since

$2.3^{r} \equiv 1(\bmod p)$ for some $r \geq i \Leftrightarrow 2.3^{r} .3^{r} \equiv 3^{r}\left(\bmod 3^{i} p\right)$ as $\left(3^{r}, 3^{i} p\right)=3^{i}, r \geq i$

then

$$
e^{2}=\left(3^{r}(1+l)\right)^{2}=2.3^{2 r}(1+l)=2.3^{r} \cdot 3^{r}(1+l) \equiv 3^{r}(1+l)\left(\bmod 3^{i} p\right),
$$

so $e^{2}=e$.

Example 3.14. The loop ring $Z_{3^{2} .5}\left[L_{n}(m)\right]$ has an idempotent $e=3^{5}(1+l)$ where $r=5,5 \mid 2.3^{5}-1$ and $l \in L_{n}(m)$.

Proposition 3.15. Let $Z_{3^{i} p}\left[L_{n}(m)\right]$ be a loop ring where $p$ is an odd prime such that $p \mid 2.3^{r}-1$ for some $r \geq i$, then there exist commuting regular elements $a, b \in Z_{3^{i} p}\left[L_{n}(m)\right]$ such that $a \neq b$.

Proof. Suppose that $p \mid 2.3^{r}-1$ for some $r \geq i$ and $a=3^{r}(1+l), b=$ $\left(3^{i} p-3^{r}\right)(1+l) \in Z_{3^{i} p}\left[L_{n}(m)\right]$. Since $2.3^{r} \cdot 3^{r} \equiv 3^{r}\left(\bmod 3^{i} p\right)$ as $\left(3^{r}, 3^{i} p\right)=3^{i}$, $r \geq i, a^{2}=a$ by the Proposition 3.13. Similarly,

$$
b^{2}=a \text { and } a b=b a=b
$$

Therefore

$$
a b=(b a) b(b a)
$$

Example 3.16. The loop ring $Z_{3^{2} .5}\left[L_{n}(m)\right]$ have commuting regular elements $a=3^{5}(1+l)$ and $b=\left(3^{2} .5-3^{5}\right)(1+l)$ where $r=5,5 \mid 2.3^{5}-1$ and $l \in L_{n}(m)$.

Proposition 3.17. Let $Z_{p_{1}^{i} p_{2}}\left[L_{n}(m)\right]$ be a loop ring where $p_{1}$ and $p_{2}$ are distinct odd primes and $p_{2} \mid 2 . p_{1}^{r}-1$ for some $r \geq i$, then there exists an idempotent element $e \in Z_{p_{1}^{i} p_{2}}\left[L_{n}(m)\right]$ such that $e \neq 0,1$. 
Proof. Suppose that $p_{2} \mid 2 . p_{1}^{r}-1$ for some $r \geq i$ and $e=p_{1}^{r}(1+l) \in$ $Z_{p_{1}^{i} p_{2}}\left[L_{n}(m)\right]$. Since

$2 . p_{1}^{r} \equiv 1\left(\bmod p_{2}\right)$ for some $r \geq i \Leftrightarrow 2 . p_{1}^{r} \cdot p_{1}^{r} \equiv p_{1}^{r}\left(\bmod p_{1}^{i} p_{2}\right)$ as $\left(p_{1}^{r}, p_{1}^{i} p_{2}\right)=p_{1}^{i}, r \geq i$

then

$$
e^{2}=\left(p_{1}^{r}(1+l)\right)^{2}=2 \cdot p_{1}^{2 r}(1+l)=2 \cdot p_{1}^{r} \cdot p_{1}^{r}(1+l) \equiv p_{1}^{r}(1+l)\left(\bmod p_{1}^{i} p_{2}\right) .
$$

So $e^{2}=e$.

Proposition 3.18. Let $Z_{p_{1}^{i} p_{2}}\left[L_{n}(m)\right]$ be a loop ring where $p_{1}$ and $p_{2}$ are distinct odd primes and $p_{2} \mid 2 . p_{1}^{r}-1$ for some $r \geq i$, then there exist commuting regular elements $a, b \in Z_{p_{1}^{i} p_{2}}\left[L_{n}(m)\right]$ such that $a \neq b$.

Proof. Suppose that $p_{2} \mid 2 \cdot p_{1}^{r}-1$ for some $r \geq i$ and $a=p_{1}^{r}(1+l), b=\left(p_{1}^{i} p_{2}-\right.$ $\left.p_{1}^{r}\right)(1+l) \in Z_{p_{1}^{i} p_{2}}\left[L_{n}(m)\right]$. Since $2 \cdot p_{1}^{r} \cdot p_{1}^{r} \equiv p_{1}^{r}\left(\bmod p_{1}^{i} p_{2}\right) \quad$ as $\quad\left(p_{1}^{r}, p_{1}^{i} p_{2}\right)=p_{1}^{i}$, $r \geq i, a^{2}=a$ by the Proposition 3.18. Similarly,

$$
b^{2}=a \text { and } a b=b a=b .
$$

Therefore

$$
a b=(b a) b(b a)
$$

\section{References}

[1] B. David, Elementary number theory, Universal Book Stall, New Delhi, 1998.

[2] H. Doostie, L. Pourfaraj, On the minimal ideals of commuting regular rings and semigroups, Internat. J. Appl. Math. 19, NO. 2 (2006), 201216 .

[3] J. M. Howie, An introduction to semigroup theory, Academic Press, London, 1976.

[4] P. Ribenboim, Rings and modules, Intersience tracts in pure and applied mathematics, John Wiley and Sons Inc, 1969.

[5] S. V. Singh, On a new class of loop rings, Phd Thesis, IIT Madras, 1994.

[6] Amir. H. Yamini, Sh. A. Safari Sabet, Commuting regular rings, Internat. J. Appl. Math. 14, NO. 4 (2003), 3557-3364.

Received: March 15, 2007 